\title{
Possibility of a Sonofusion Reactor - Nuclear Fusion induced by Cherenkov Radiation from the ZPF field in a Collapsing Water Bubble
}

\section{Takaaki Musha ${ }^{1,2^{*}}$ and Gary Stephenson ${ }^{3}$}

\author{
${ }^{1}$ Advanced Science - Technology Research Organization, Yokohama, Japan \\ ${ }^{2}$ Foundation of Physics Research Center (FoPRC), Cosenza, Italy \\ ${ }^{3}$ Seculine Consulting, California, USA.
}

*Corresponding author details: Takaaki Musha; takaaki.mushya@gmail.com

\begin{abstract}
Sonofusion, also known as bubble fusion, is the name for a nuclear fusion reaction hypothesized to occur during a high-pressure version of sonoluminescence. By applying this phenomenon, the researchers caused bubbles in the deuterated acetone liquid with oscillating sound waves to expand and then violently collapse for producing a flash and enormous heat that has the potential to fuse nuclei together. In this paper, the authors present the theoretical explanation of sonoluminescence, which accounts for the sonofusion induced by Cherenkov radiation which is generated from tachyon pairs created from ZPF field in a collapsing water bubble, and the possible structure of a sonofusion reactor.
\end{abstract}

\section{Keywords: sonofusion; sonoluminescence; Casimir energy; Cherenkov radiation; tachyon; zero-point energy; energy creation}

\section{INTRODUCTION}

Sonofusion or bubble fusion is the common name for a nuclear fusion reaction hypothesized to occur during a high-pressure version of sonoluminescence, an extreme form of acoustic cavitation. This reaction is termed acoustic inertial confinement fusion since the inertia of the collapsing bubble wall confines the energy, causing an extreme rise in temperature. The high temperatures that sonoluminescence can produce raise the possibility to achieve thermonuclear fusion in the deuterated liquid and the researchers caused bubbles in the deuterated acetone $\left(\mathrm{C}_{3} \mathrm{D}_{6} \mathrm{O}\right)$ to expand and then violently collapse to generate extremely high temperature to produce a shock wave that has the potential to fuse nuclei together, which leads to the productions of neutrons. This phenomena were first reported by R.P. Taleyarkhan and his colleagues at the Oak Ridge National Laboratory (ORNL) in the journal, Science [1], that acoustic cavitation experiments conducted with deuterated acetone showed measurements of tritium and neutron output that were consistent with the occurrence of fusion. The neutron emission was also reported to be coincident with the sonoluminescence pulse, which suggests that its source was fusion caused by the sonoluminescence [2]. Shock wave simulations seem to indicate that the temperatures inside the collapsing bubbles may reach up to 10 megakelvins, i.e. as hot as the center of the Sun. The neutron emission was also reported to be coincident with the sonoluminescence pulse, a key indicator that its source was fusion caused by the heat and pressure inside the collapsing bubbles. In theory, the vibrations collapsed gas bubbles in the solvent, heating them to temperatures high enough to fuse hydrogen atoms and release energy.
However, the mechanism of the phenomenon of sonoluminescence remains unsettled and theories which included hotspot, bremsstrahlung radiation, collisioninduced radiation and corona discharges, nonclassical light, proton tunneling, electrodynamic jets, fractoluminescent jets, and so forth were presented [3].

In this paper, we show the possibility to make a fusion reaction theoretically by the Cherenkov radiation generated inside the collapsing bubbles.

\section{THEORETICAL ANALYSIS}

Sonoluminescence (SL) is light produced from the collapsing bubble in water under ultrasound. Sonoluminescence can occur when a sound wave of sufficient intensity induces a gaseous cavity within a liquid to collapse quickly. The bubble reaches a maximum at about ten times its original size, then it completely collapses within a few milliseconds later, emitting a burst of light each time when it collapses. The experimental results obtained in various fluids have shown that a flash of light emitted from the collapsing bubble with a maximum diameter of 100 microns that creates a temperature of $5500{ }^{\circ} \mathrm{C}$ with a blackbody spectrum when it rapidly shrinks to less than one micron in radius. They have also shown that light flashes from the bubbles are extremely short persisted only for 50 picoseconds or shorter with peak intensities of the order of 1 10 mW, which was too brief for the light to be produced by some atomic process. For explaining these properties of sonoluminescence, Schwinger wrote papers wherein the Casimir energy released in the collapse of a spherically symmetric bubble or a cavity with a volume $V$ in a dielectric fluid can be given by [4] 


$$
\begin{aligned}
E_{\text {cavity }} & =2 V \int_{0}^{k} \frac{4 \pi k^{2} d k}{(2 \pi)^{3}} \frac{\hbar c k}{2}\left(\frac{1}{\sqrt{\varepsilon_{\text {in }}}}-\frac{1}{\sqrt{\varepsilon_{\text {out }}}}\right)+\cdots \\
& =\frac{1}{6 \pi} \hbar c R^{3} K^{4}\left(\frac{1}{\sqrt{\varepsilon_{\text {in }}}}-\frac{1}{\sqrt{\varepsilon_{\text {out }}}}\right)+\cdots,
\end{aligned}
$$

where $V$ is the volume of a bubble, $R$ is its radius, $K$ is a high wavenumber cutoff that characterizes the wavenumber at which the dielectric constants drop to their vacuum values, $\hbar$ is a Plank's constant divided by $2 \pi, c$ is a light speed, and $\varepsilon_{\text {in }}$ and $\varepsilon_{\text {out }}$ are dielectric constants for outside the bubble and inside it respectively.

On the basis of his theory, Liberati, Visser, Belgiorno and Sciama derived the equation in the framework of the dynamical Casimir effect to give reasonable agreement with observations, which included the contribution of dynamical Casimir effect shown as [5]

$$
\begin{aligned}
E & =\frac{1}{8 \pi n^{2} n^{\prime}}\left(\frac{n-n^{\prime}}{n n^{\prime}}\right)^{2} \hbar c K(R K)^{3} \\
& \approx \frac{1}{8 \pi n^{2}}\left(1-\frac{1}{n}\right)^{2} \hbar c R^{3} K^{4}
\end{aligned}
$$

where $n$ is a refractive index of liquid and $n^{\prime}$ is a refractive index of gas inside the bubble. However this mechanism cannot explain impossibly short time scales of SL radiation because it is required that the overall collapse time of a bubble is $10^{-4}$ sec instead of the impossible short time scale on the order of $10^{-15}$ sec.

Thus, the authors have proposed another missing component of sonoluminescence due to Cherenkov radiation from tachyon pairs generated from the ZPF (zero-point fluctuation) field contained in a collapsing bubble instead of a conventional theory for explaining sonoluminescence as a QED vacuum effect, given as follows.

\section{Cherenkov Radiation from Tachyon Pairs Created in a ZPF Field}

T.Musha, one of the authors, proposed the possibility in his paper that the Cherenkov radiation can be generated from tachyon pairs created in a collapsing bubble shown as follows [6];

From the wave equation taking account of the special relativity (i.e. Klein-Gordon equation) given by

$$
i \hbar \frac{\partial}{\partial t} \psi=H \psi
$$

where $H=\sqrt{p^{2} c^{2}+M^{2} c^{4}}$ ( $p$ : momentum of the particle, $M$ : effective mass) and $\psi$ is a wave function for the particle, the following equation can be obtained for the accelerated particle shown as

$$
\frac{\partial \psi}{\partial p}=-\frac{i}{M a \hbar} \sqrt{p^{2} c^{2}+M^{2}} c^{4} \psi
$$

where $a$ is a proper acceleration of the virtual elementary particle generated from a ZPF field. If the photon is generated in a quantum region, which size is $l$, the proper acceleration of the photon becomes

$$
a=\frac{1}{m} \frac{\Delta p}{\Delta t} \approx \frac{c^{2}}{l},
$$

from the uncertainty of momentum and energy given by $\Delta p \cdot l \approx \hbar$ and $\Delta E=\Delta p \cdot c$, respectively, when we let $\Delta E=m c^{2}$ and $\Delta t=l / c$.

From which, it can be seen that there is possibility for the accelerated particle which can tunnel through the light barrier. According to the WKB approximation, the penetration probability through the light barrier of the tunneling photon can be estimated by [7]

$T_{p} \approx\left|\psi_{*}\right|^{2} /|\psi|^{2}$

$=\exp \left[-\frac{\omega}{c} l \sqrt{\beta^{2}-1}\left(\frac{\beta}{\beta^{2}-1}-\log (\hbar \omega / C)-\log (\mathrm{l}+\beta)\right)\right]$

where $\beta$ is the ratio of the particle velocity divided by the light speed.

By applying the uncertainty principle to the virtual superluminal particle, we obtain $\beta \approx 2$ [8], then Eq. (6) becomes

$$
T_{p} \approx \exp \left[-\frac{\omega}{c} l \sqrt{3}\left(\frac{2}{3}-\log (\hbar \omega / c)-\log 3\right)\right],
$$

Supposing that $l$ has a size of the Plank length, then Eq. (7) can be approximated by

$$
T_{p}(\omega) \approx \exp \left[-\gamma \cdot l_{p} \omega\right]
$$

where $l_{p}$ is a Plank length and

$$
\gamma=\frac{3 \log 3-2+3 \log (\hbar / c)}{\sqrt{3} c} \approx 5.62 \times 10^{-7} .
$$

If tachyon pairs created from the ZPF field have an electric charge, it radiates photons at the angle of $\theta=\cos ^{-1}[1 /(\beta n)]$, where $\theta$ is half-angle of the Cherenkov radiation from the particle moving at the speed of $\beta=v / c$ and $n$ is the index of refraction of surrounding medium.

As the radiation field by the Cherenkov effect can be regarded as a thermal equilibrium system filled with nonradiating electromagnetic waves, it is permitted that small fraction of energy from non-radiating electromagnetic field can be radiated as blackbody radiation according to the SED theory shown as follows;

$$
\begin{aligned}
\rho_{E}(\omega)= & \frac{\hbar \omega^{3}}{2 \pi c^{3}} T_{p}(\omega) \frac{\sum_{k=0}^{\infty} k e^{-k \hbar \omega / k_{B} T}}{\sum_{k=0}^{\infty} e^{-k \hbar \omega / k_{B} T}} \\
& =\frac{\hbar \omega^{3}}{2 \pi^{2} c^{3}} \exp \left(-\gamma l_{p} \omega\right)\left[\exp \left(\frac{\hbar \omega}{k_{B} T}-1\right)\right]^{-1}
\end{aligned}
$$

where $k_{B}$ is the Boltzmann constant and $T$ is the absolute temperature of radiation.

From the calculation by Liberati et al [5], the total number of created photons by Casimir effect confined in a bubble becomes 


$$
N \approx \frac{1}{6 \pi n n^{\prime}}\left(\frac{n-n^{\prime}}{n n^{\prime}}\right)^{2}(R K)^{3} .
$$

Thus, the average energy per emitted photon can be given by

$$
<E>\approx E / N=\frac{3}{4} \hbar c K / n \text {. }
$$

From which, the temperature inside the collapsing bubble due to the Casimir effect can be obtained from

$$
<E>\approx \frac{3 \pi}{2} \frac{\hbar c}{n \lambda}=k_{B} T,
$$

where $\lambda$ is a wavelength of ultraviolet cutoff frequency given by $\lambda=2 \pi / K$.

Hence the vacuum inside the collapsing bubble attains the high temperature due to the Casimir effect and it enhances Cherenkov radiation to convert vacuum energy into heat shown as follows;

By the temperature given by Eq. (13), the energy density of Cherenkov radiation from tachyon pairs created from the ZPF field at the temperature generated by the Casimir effect becomes

$$
\rho_{E}(\omega)=\frac{\hbar \omega^{3}}{\pi^{2} c^{3}} \exp \left(-\gamma l_{p} \omega\right) \cdot\left[\exp \left(\frac{2 n \lambda}{3 \pi c} \omega\right)-1\right]^{-1}
$$

From the assumption that the collapsing bubble is black, i.e. it perfectly absorbs all wavelength of electromagnetic radiation that requires the mean free path of photons be much smaller than the size of the bubble[9], the bubble radiates all electromagnetic energy when it shrinks to the size less than the mean free path $\bar{l}$, which can be defined by $\bar{l}=(\kappa \rho)^{-1}$, where $\kappa$ is an absorption coefficient and $\rho$ is a density of gas.

Then the total energy radiated by Cherenkov radiation becomes

$$
E_{c}=V \int_{0}^{\infty} \rho_{E}(\omega) d \omega
$$

$=\frac{4}{3} \pi\left(R_{0}^{3}-R_{m}^{3}\right) \times \frac{\hbar}{\pi^{2} c^{3}} \int_{0}^{\infty} \omega^{3} \exp \left(-\gamma l_{p} \omega\right)\left[\exp \left(\frac{2 n \lambda}{3 \pi c} \omega\right)-1\right]^{-1} d \omega$

$=\frac{81 \pi^{3}}{2} \frac{\hbar c}{n^{4} \lambda^{4}}\left(R_{0}^{3}-R_{\min }^{3}\right) \zeta(4,1+\alpha)$,

where $\alpha=3 \pi l_{p} \gamma c /(2 n \lambda), R_{0}$ is the radius of a bubble at the beginning of a rapid collapse phase with no electromagnetic energy is released from the bubble, $R_{\text {min }}$ is a minimum radius of the bubble when it radiates all electromagnetic energy, and $\zeta(m, n)$ is a Hurwitz zeta function [10].

The process of a collapsing bubble that radiates strong light flash, which consists of phases, such as (1) slow expansion, (2) turnaround at maximum radius, (3) collapse with moderate speed, (4) rapid collapse that the heat can no longer escape from the bubble, and (5) emission of light [11].

Inside a collapsing bubble, as the hot plasma created by collisions of the atoms and molecules with high temperature makes the mean free path of photons much shorter to be $R_{\min } \approx 0$, the maximum energy emitted by the Cherenkov radiation from virtual tachyon pairs in a bubble, which is due to the confined thermal radiation of electromagnetic energy created by the Casimir effect inside the collapsing bubble, can be roughly estimated as

$$
E \approx \frac{81}{32 \pi} \frac{\hbar c}{n^{4}} R_{0}^{3} K^{4} \zeta(4,1+\alpha) .
$$

From the uncertainty principle, the path of FTL (fasterthan-light) photons created from the ZPF of electromagnetic field can be estimated by $l \approx c / \omega[10]$, from which we have $l \approx 0.4 \sim 0.8 \mu m$ for the visible spectrum of the emitted light.

Assuming that the path of the FTL photon almost equals to the free pass of photons created inside the bubble, it is considered that electromagnetic energy confined in a collapsing bubble is radiated when it reaches the size less than one micron, which can explain the experimental result that addition of small amount of noble gas increases the intensity of emitted light because the free pass of photons becomes shorter by the formation of a hot plasma. This mechanism of creating photons can also explain the short time scale of the radiation from the bubble because the heat can no longer escape from the bubble until it reaches the size of the path of FTL photons.

\section{Possibility of Sonofusion to create Energy in Collapsing Bubbles}

Critical ignition temperature for fusion to produce nuclear strong force to overcome the coulomb repulsion can be estimated as $4 \times 10^{8}{ }^{\circ} \mathrm{K}$ (deuterium-deuterium) and $4.5 \times 10^{7}{ }^{\circ} K$ (deuterium-tritium), in order to achieve reasonable rates of energy production. It can be estimated for the bubble with the size of $R_{\max }=40 \mu m$ that the radiation temperature from it can attains about $10^{11}{ }^{\circ} \mathrm{K}$ from Eqs. (13) and (16).

This temperature is enough to induce a D-D reaction of the deuterium shown as;

$$
\mathrm{D}+\mathrm{D} \rightarrow{ }^{3} \mathrm{He}+\mathrm{n}(2.5 \mathrm{MeV})
$$

Thus, it can be considered that the fusion temperature can be obtained by Cherenkov radiation created from ZPF field inside the collapsing bubbles.

Figure.1 shows the process of generation of energy by using sonofusion, which is powered by the Cherenkov radiation created from the ZPF field in collapsing bubbles. The process of sonofusion consists of phases, (1) cavitation creates bubbles, (2a) lower acoustic driving pressure collapse bubbles, (2b) ZPF mode loss triggers virtual photons, (2c) virtual photon driven tachyonic field develops, (2d) tachyonic condensation via Cherenkov radiation, $(2 \mathrm{e})$ confined Cherenkov radiation produces fusion, (3) vaporized ions undergo D-D fusion and (4) heat is coupled to power generation.

Hence, by bombarding a water including atoms of deuterium with powerful oscillating sound waves generated by PZTs, we can obtain the fusion energy from the apparatus containing deuterated liquid. 


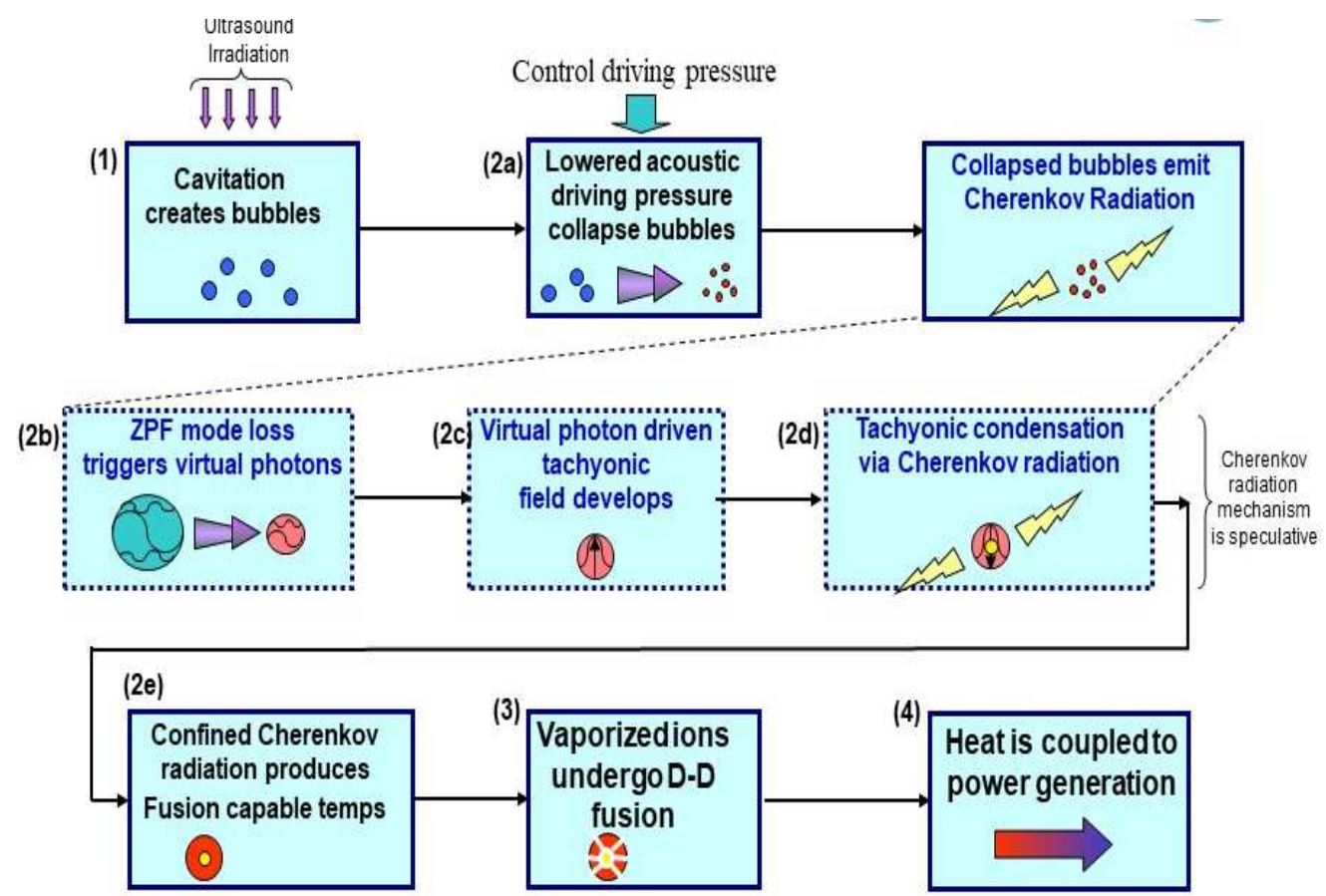

FIGURE 1: Process of the energy creation by using sonofusion

Sea water contains 6 grams of deuterium per 200 liters inside it. One-gram deuterium can generate the heat equivalent to $8000 \mathrm{Kg}$ of oil.

Hence 1000 liters of sea water can generate the heat about 900MWh by sonofusion

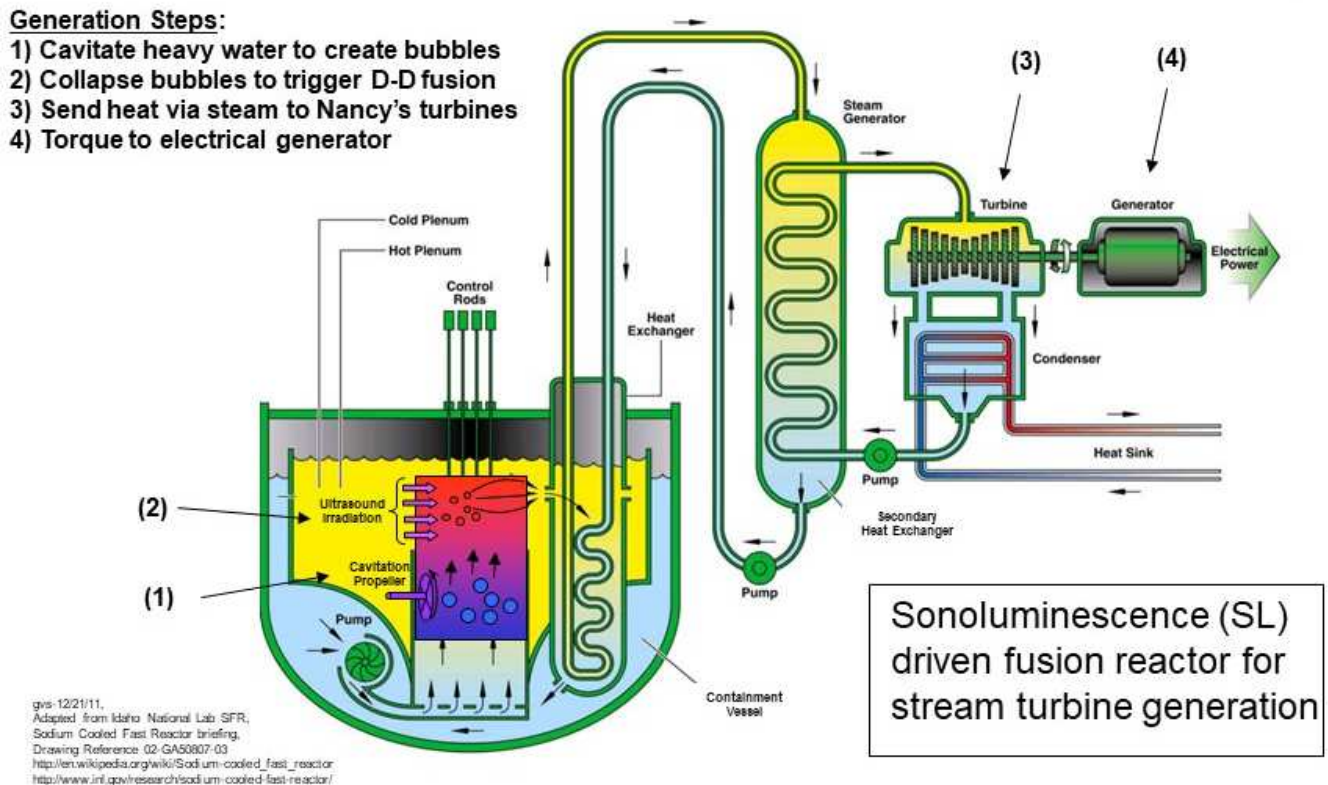

FIGURE 2: Conceptual diagram of a sonofusion reactor

This generated heat is enough to propel the conventional submarines in the sea.

Figure.2 is the diagram for the steam turbine generator using a sonofusion reactor to propel the ship.
Current generations of nuclear submarines need to be refueled throughout their 25-year lifespans and there is a problem of radioactive waste. But the large amount of power generated by a sonofusion reactor allows submarines to operate at high speed for long periods of time without radioactive contamination. Hence, we can construct a super submarine to cruise around the world without refueling. 


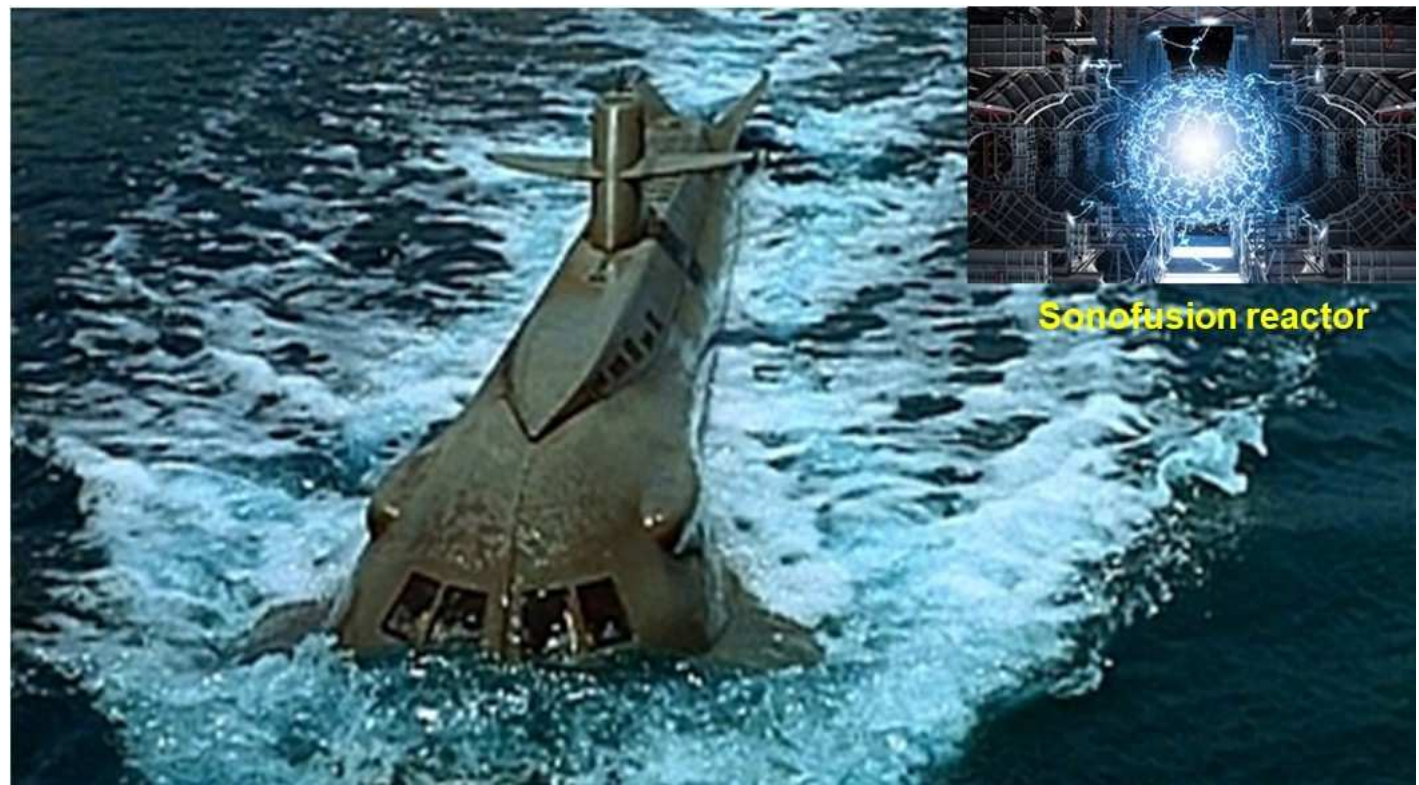

FIGURE 3: Conceptual figure of the future submarine using a sonofusion reactor

\section{CONCLUSION}

From the theoretical analysis, it can be seen that the Cherenkov radiation from the ZPF field in a collapsing bubble can create energy enough to induce a nuclear fusion by its large amount of heat, which can achieve thermonuclear fusion during cavitation of the deuterated liquid, that may lead to the possible source of unlimited energy for submarines and other vehicles in the future.

\section{REFERENCES}

[1] R.P.Taleyarkhan, C.D.West, J.S.Cho, R.T.Lahey Jr., R.I.Nigmatulin and R.C.Block, Evidence for Nuclear Emissions During Acoustic Cavitation, Science,Vol.295(2002)1868-1873.

[2] R.P.Taleyarkhan, C.D.West, J.S.Cho, R.T.Lahey Jr., R.I.Nigmatulin and R.C.Block Additional evidence of nuclar emissions during acoustic cavitation, Physical Review E. 69 (2004)036109,1-11.

[3] B.R.Barber, R.A.Hiller, R.Lofstedt, S.J.Putterman and K.R.Wenigner, Defining the Unknown Sonoluminescence, Physics Reports, Vol.281, No.12 (1997) 65-143.

[4] J. Schwinger, Casimir energy for dielectrics: spherical geometry, Proc.Natl.Acad. Sci.USA 89,11(1992) 1111811120; Casimir light: the source. Proc.Natl.Acad.Sci.USA, 90(1993) 2105-2106.
[5] S.Liberati, M.Visser, F.Belgiornoss and D.W.Sciama, Sonoluminescence as a QED vacuum effect: probing Schwinger's proposal, J.Physcs. A: Math. Gen.33 (2000) 2251-2272.

[6] T.Musha, Superluminal Effect for Quantum Computation that Utilizes Tunneling Photons, Physics Essays, Vol.18, No.4 (2005) 525-529.

[7] T.Musha, Thermal Radiation Generated inside the Sun due to the Cherenkov Radiation from ZPF Field, Far East Journal of Applied Mathematics, Vol.37, Issue.2 (2009)229-235.

[8] T.Musha, Cherenkov Radiation from Faster-ThanLight Photons Created in a ZPF Background, Journal of Theoretics,Vol.3,No.3 (2001).

[9] M.P.Brenner, Single-bubble sonoluminescence, Review of Modern Physics, Vol.74, No.2 (2002) 425-484.

[10] T.Musha, L.M.Caligiuri, Energy Creation by Cherenkov Radiation from the ZPF field in a Water Bubble, Journal of Scientific and Engineering Research, 7(6),(2020) 167-174.

[11] S.J. Putterman and K.R.Weninger, Sonoluminescence: How Bubbles Turn Sound into light, Ann. Rev. Fluid Mech.32 (2000)445-476. 